\title{
The SOC1-like gene BoMADS50 is associated with the flowering of Bambusa oldhamii
}

\author{
Dan Hou', Ling Li', Tengfei Ma', Jialong Pei', Zhongyu Zhao', Mengzhu Lu', Aimin Wu ${ }^{2,3}$ and Xinchun Lin ${ }^{1}$
}

\begin{abstract}
Bamboo is known for its edible shoots and beautiful texture and has considerable economic and ornamental value. Unique among traditional flowering plants, many bamboo plants undergo extensive synchronized flowering followed by large-scale death, seriously affecting the productivity and application of bamboo forests. To date, the molecular mechanism of bamboo flowering characteristics has remained unknown. In this study, a SUPPRESSOR OF OVEREXPRESSION OF CONSTANS1 (SOC1)-like gene, BOMADS50, was identified from Bambusa oldhamii. BOMADS50 was highly expressed in mature leaves and the floral primordium formation period during B. oldhamii flowering and overexpression of BOMADS50 caused early flowering in transgenic rice. Moreover, BoMADS50 could interact with APETALA1/FRUITFULL (AP1/FUL)-like proteins (BoMADS14-1/2, BoMADS15-1/2) in vivo, and the expression of BOMADS50 was significantly promoted by BOMADS14-1, further indicating a synergistic effect between BoMADS50 and BoAP1/FUL-like proteins in regulating B. oldhamii flowering. We also identified four additional transcripts of BoMADS50 (BOMADS50-1/2/3/4) with different nucleotide variations. Although the protein-CDS were polymorphic, they had flowering activation functions similar to those of BOMADS50. Yeast one-hybrid and transient expression assays subsequently showed that both BOMADS50 and BOMADS50-1 bind to the promoter fragment of itself and the SHORT VEGETATIVE PHASE (SVP)-like gene BOSVP, but only BOMADS50-1 can positively induce their transcription. Therefore, nucleotide variations likely endow BOMADS50-1 with strong regulatory activity. Thus, BOMADS50 and BOMADS50-1/2/3/ 4 are probably important positive flowering regulators in B. oldhamii. Moreover, the functional conservatism and specificity of BOMADS50 and BOMADS50-1 might be related to the synchronized and sporadic flowering characteristics of B. oldhamii.
\end{abstract}

Woody bamboos are tree-like woody grasses and serve as an economically important resource worldwide because they produce timber, fiber, food, and other products ${ }^{1}$. Among these, Bambusa oldhamii is famous for its delicious and nutritious shoots. This plant is highly adaptive and shows strong regeneration, and the shooting period can last for 5 months per year. The annual output of $B$. oldhamii shoots reaches up to 10,000 tons, which have

\footnotetext{
Correspondence: Aimin Wu (wuaimin@scau.edu.cn) or

Xinchun Lin (linxcx@163.com)

${ }^{1}$ State Key Laboratory of Subtropical Silviculture, Zhejiang A \& F University,

Lin'an, 311300 Hangzhou, China

${ }^{2}$ State Key Laboratory for Conservation and Utilization of Subtropical Agrobioresources, South China Agricultural University, 510642 Guangzhou, China Full list of author information is available at the end of the article

These authors contributed equally: Dan Hou, Ling Li
}

significant economic value. Bamboo has a unique flowering characteristic and unpredictable vegetative period ${ }^{2}$. These plants generally die after blooming, resulting in reduced production or even the death of shoots, which seriously affects the productivity, development, and ecosystem of bamboo forests. Consequently, increasing attention has been given to bamboo flowering for its scientific importance and crucial role in some human communities.

The flowering cycles of different bamboo species vary. For example, the flowering time of Phyllostachys is $13-120$ years, while that of Bambusa is $30-150+$ years $^{3-5}$. Interestingly, although the flowering of bamboo has a strong periodicity, some bamboo species have flowering habits that are extensively synchronized and sporadic ${ }^{4,6}$.

\section{(c) The Author(s) 2021}

(c) (i) Open Access This article is licensed under a Creative Commons Attribution 4.0 International License, which permits use, sharing, adaptation, distribution and reproduction cc) in any medium or format, as long as you give appropriate credit to the original author(s) and the source, provide a link to the Creative Commons license, and indicate if changes were made. The images or other third party material in this article are included in the article's Creative Commons license, unless indicated otherwise in a credit line to the material. If material is not included in the article's Creative Commons license and your intended use is not permitted by statutory regulation or exceeds the permitted use, you will need to obtain permission directly from the copyright holder. To view a copy of this license, visit http://creativecommons.org/licenses/by/4.0/. 
For example, the majority of $B$. oldhamii do not flower until $\sim 30-60$ years of age ${ }^{7}$, but even in a bamboo population formed by the same clone, some clusters or branches frequently blossom sporadically ${ }^{4,8}$. Many hypotheses about the bamboo flowering cycle have emerged ${ }^{3,4,9}$. In recent years, the completion of genome sequencing of Phyllostachys edulis ${ }^{10,11}$, transcriptome sequencing of different bamboo species ${ }^{12}$, and functional analysis of bamboo flowering genes ${ }^{7,13}$ have provided a crucial basis for bamboo flowering studies. However, until now, "the molecular aspects of bamboo flowering remain at a nascent stage"14, and why many bamboo species bloom periodically and sporadically is still unclear.

MADS transcription factors are best known for their involvement in determining floral development ${ }^{15-18}$. In addition to their functional roles as floral organ identity genes, $M A D S$ genes also participate in floral induction ${ }^{19-21}$. In Arabidopsis thaliana, SUPPRESSOR OF OVEREXPRESSION OF CONSTANS1/AGAMOUS-LIKE 20 (SOC1/AGL20) belongs to the MIKC (MADS DNAbinding domain, intervening domain, keratin-like domain, and C-terminal domain)-type MADS-box gene family ${ }^{22}$, constitutes a major hub in a complex regulatory network underlying floral timing and plays a central role in $A$. thaliana reproduction by integrating signals of the photoperiod, autonomous, vernalization, age, and gibberellin pathways $^{23,24}$. The SOC1-like gene also exists as a flowering activator in many plant species, including Zea mays ${ }^{25}$, Medicago truncatula ${ }^{26}$, and Eriobotrya japonica ${ }^{27}$. In rice, OsMADS50, a gene highly homologous to A. thaliana $S O C 1$, functions as a long-day (LD)-specific flowering promoter mainly through the Oryza sativa LEC2 and FUSCA3 Like 1-Early heading date 1-Heading date 3a/ RICE FLOWERING LOCUS T1 (OsLFL1-Ehd1-Hd3a/ RFT1) pathways ${ }^{28,29}$. This gene may be regulated by the $F L O / L F Y$ homolog of rice (RFL) and function parallel to other rice flowering time genes, such as Heading date 1 (Hd1) and GIGANTEA (GI), to eventually activate the expression of the FLOWERING LOCUS T (FT)-like gene ${ }^{30}$. Therefore, "a proper integration of inductive floral cues by SOC1-like genes is an important step in regulating plants floral induction" 26 .

In woody bamboos, the SOC1-like gene is considered one of the potential factors influencing vegetative growth and floral timing ${ }^{2}$. This finding is mainly because the protein structures of woody bamboo SOC1-like proteins are clearly different from those of herbaceous plants (with annual flowering behavior, such as rice and other typical grasses), which may lead to the loss/malfunction of SOC1like genes and even strong variations in flowering time ${ }^{2}$. Additionally, the overexpression of the SOC1-like genes PvSOC1 and PvMADS56 from $P$. violascens can significantly promote flowering in transgenic $A$. thaliana or rice, indicating their essential roles in bamboo flowering determination ${ }^{31,32}$. Therefore, understanding the function of the SOC1-like gene is necessary for the elucidation of the flowering mechanism in woody bamboo.

In the current study, we reported the nuclear-localized SOC1-like protein BoMADS50 from B. oldhamii. BoMADS50 is likely involved in bamboo flowering induction by acting in coordination with BoAP1/FUL proteins. Moreover, the other four BoMADS50 transcripts (BoMADS50-1/2/3/4) with different nucleotide variations might be functionally conserved as flowering activators. However, unlike BoMADS50, BoMADS50-1 showed strong regulatory activity toward target genes. In brief, our data define a putative regulatory pathway of BoMADS50 and provide a new scientific hypothesis for massive bamboo and sporadic flowering regulation.

\section{Results}

\section{Cloning and identification of BoMADS50}

To isolate the SOC1-like gene from B. oldhamii, we cloned a cDNA fragment that showed high similarity to $S O C 1$ orthologs from various plant species. The cDNAs with the full CDS of BoMADS50 were obtained using the $3^{\prime}$ and $5^{\prime}$ RACE methods. The full-length coding sequence (CDS) region of BoMADS50 was $678 \mathrm{bp}$, encoding 225 aa. Similar to those of other known SOC1-like genes, the amino acid sequence of BoMADS50 contained a well-conserved MADS domain, a less conserved $\mathrm{K}$ domain, and a divergent $\mathrm{C}$-terminal region (Fig. 1a). Moreover, multiple sequence alignment confirmed the presence of a conserved SOC1 motif that is specific to the TM3-like (tomato MADSbox gene 3-like) clade of MADS-box genes in the BoMADS50 protein $^{22}$. BoMADS50 shared high sequence similarities with OsMADS50 in rice and SOC1-like proteins from other bamboo species, further indicating that BoMADS50 is a SOC1 homolog in $B$. oldhamii, which belongs to a SOC1-like group (Fig. 1b). Finally, as shown in Fig. 1c, the fused protein of BoMADS50-GFP was explicitly localized in the nucleus, indicating that BoMADS50 functions in the nuclei.

\section{Characteristics of $B$. oldhamii flowering and expression analysis of BoMADS50}

B. oldhamii has a mixed inflorescence with a spikelet as the basic unit ${ }^{33}$. Here, we separated spikelets into three developmental stages based on their size: floral I $(\sim 3.00 \pm 0.35-4.33 \pm 0.50 \mathrm{~mm})$, floral II $(\sim 6.69 \pm 0.71$ $-7.25 \pm 0.50 \mathrm{~mm})$, and floral III $(9.57 \pm 0.53-11.08 \pm$ $0.36 \mathrm{~mm}$ ) (Fig. 2a). At the early stage of spikelet differentiation (floral I), the apical meristem has a semiorbicular shape (Fig. 2b-i). This structure continued to elongate and enlarge and produced the protuberance, which developed into the floret primordium (Fig. 2b-ii and iii). In floral II, the floret primordium continued to 


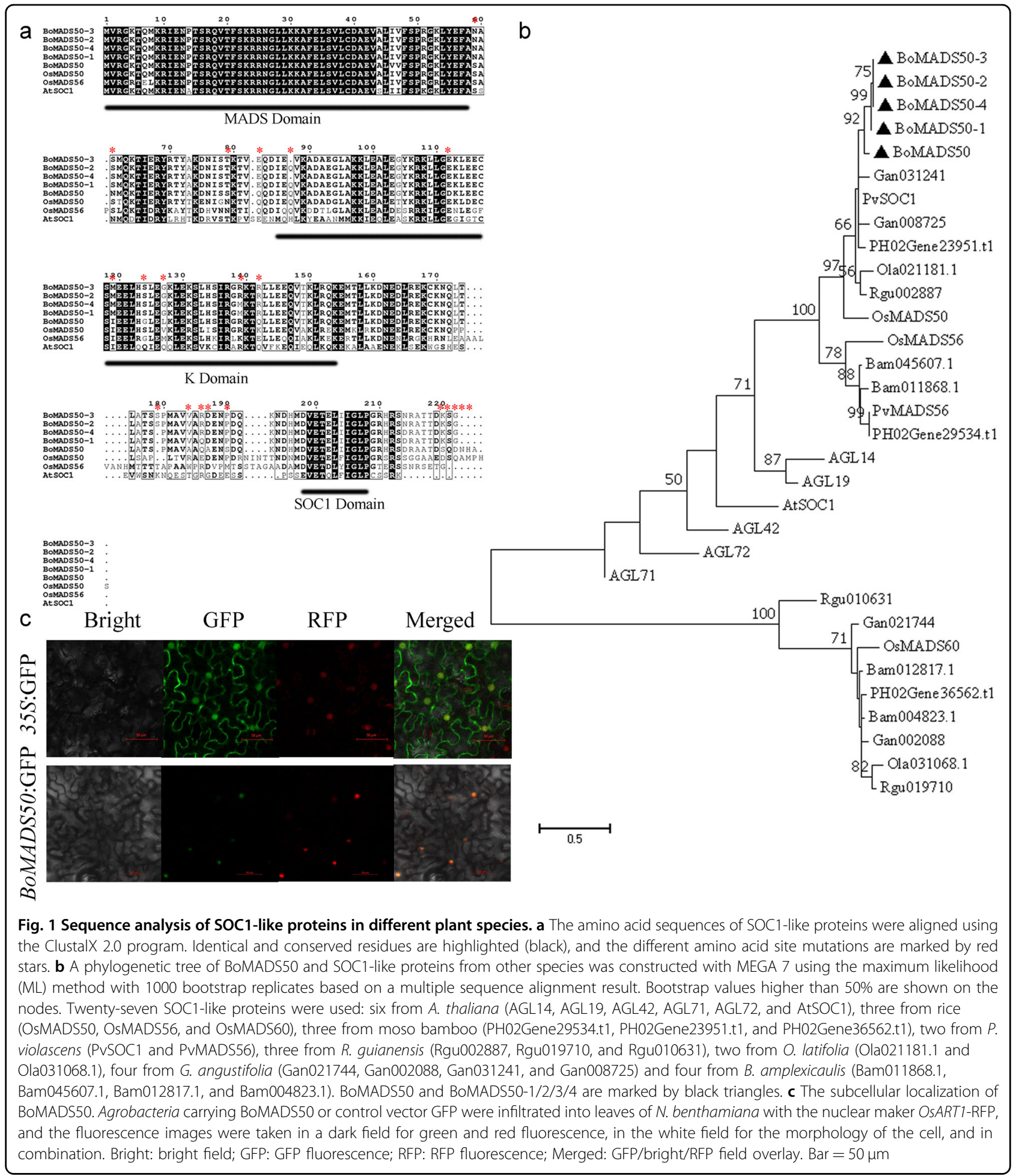

develop from the base to the top in the spikelet (Fig. 2biv). The floret at the base developed first, and then, the flower organ primordium appeared and gradually developed into the lemma, palea, lodicule, stamen, and pistil (Fig. 2b-v). At the last stage (floral III), the flower bud primordium at the top began to differentiate (Fig. $2 \mathrm{~b}$-vi). All the flower organs in the basal floret completed development and formed mature pollen grains and ovaries (Fig. 2b-vii). Thus, the floral primordium of B. oldhamii was formed as early as in the floral I stage 


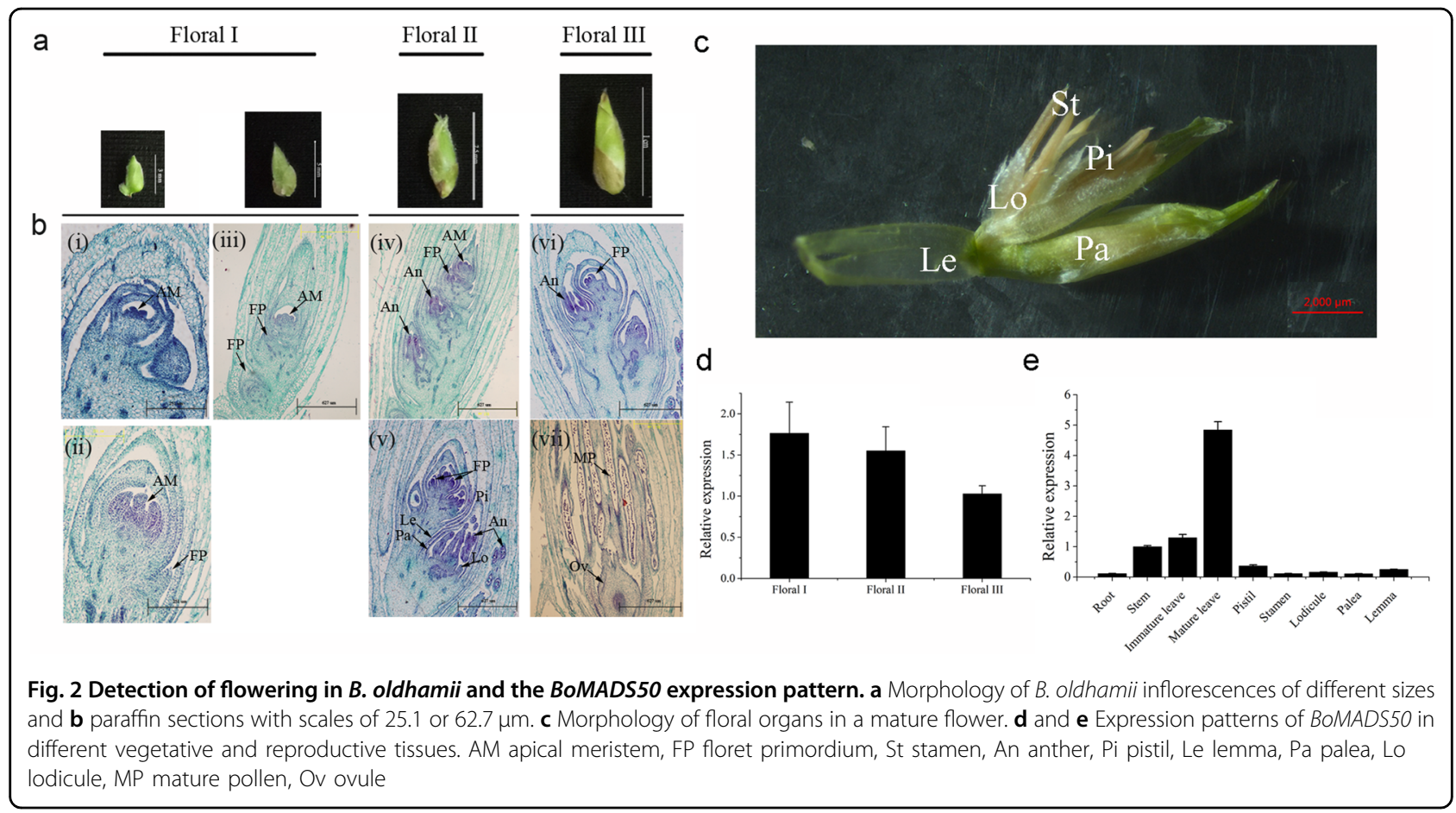

and kept forming from the base to the top in the spikelet. Mature flower organs gradually appeared in the base spikelet (floral II). When the top floret matured, the development of the spikelet ended (floral III).

The mature flowers of $B$. oldhamii had six stamens, one pistil, three lodicules, one palea, and one lemma (Fig. 2c). However, even when the flora matured, the stigma usually failed to be exposed, and the stamens were mostly withered (Fig. S1a). Many wrinkled and defective pollens formed, and two layers of anther walls were observed (Fig. S1b). These flowering characteristics and abnormal microspores are likely the main reasons for the sterility of B. oldhamii ${ }^{34}$.

To understand the putative function of BoMADS50 in flower development, we examined the expression pattern of BoMADS50 in various vegetative and reproductive organs of B. oldhamii. As shown in Fig. 2d, during in vitro flowering, BoMADS50 expression reached its highest level at the floral I stage, decreased gradually with the development of spikelets, and was lowest in the floral III stage. In the vegetative tissues, BoMADS50 was highly expressed in mature leaves, which was more than five times higher than that in roots and stems (Fig. 2e). In flower organs, the expression of BoMADS50 was barely detected. This finding indicates that BoMADS50 may integrate flowering signals from the leaves for the transition from a vegetative to a reproductive state but does not directly regulate flower organ development.
Overexpression of BoMADS50 accelerates flowering in rice

As bamboo has a closer relationship with rice, we transformed BoMADS50 into rice to determine its role in the regulation of flowering (Fig. 3). Three independent homozygous lines of BoMADS50 were selected $(O E 2 / 3 / 7$, grown under field conditions) according to different gene expression levels in the T3 generation. Compared with wild-type (WT) plants, the BoMADS50 overexpression plants showed phenotypes such as earlier flowering, smaller panicles, and fewer seeds (Fig. 3a, b). In transgenic rice, the average relative expression level of BoMADS50 was $4.95 \pm 0.95$ (Fig. 3c), and the heading time and seedling height were $\sim 51.74 \pm$ 2.65 days and $60.33 \pm 4.04 \mathrm{~cm}$, respectively, $\sim 25$ days earlier and $27 \mathrm{~cm}$ shorter than those of the WT lines $(76.62 \pm 2.02$ days and $87.22 \pm 3.39 \mathrm{~cm}$ ) (Fig. $3 \mathrm{~d}$, e). To study the molecular mechanism by which BoMADS50 promotes flowering, we analyzed the expression patterns of the critical regulators in the rice photoperiod pathway (Fig. 3f). In the BoMADS50-overexpressing plants, the expression of Early heading date2 (Ehd2), OsMADS50, RFT1, and Ehd1 was obviously upregulated, approximately $2.56 \pm 0.28,2.32 \pm 0.46,42.99 \pm$ 21.68 , and $29.27 \pm 10.29$ times higher than that in the WT plants, respectively. In addition, the expression of OsHd1, OsCO3 (a CONSTANS-LIKE gene), Ghd7 (a CO-like gene containing a CCT motif), and OsMADS56 was clearly downregulated after overexpression of BoMADS50, leading to a decrease of at least $\sim 60 \%$. 


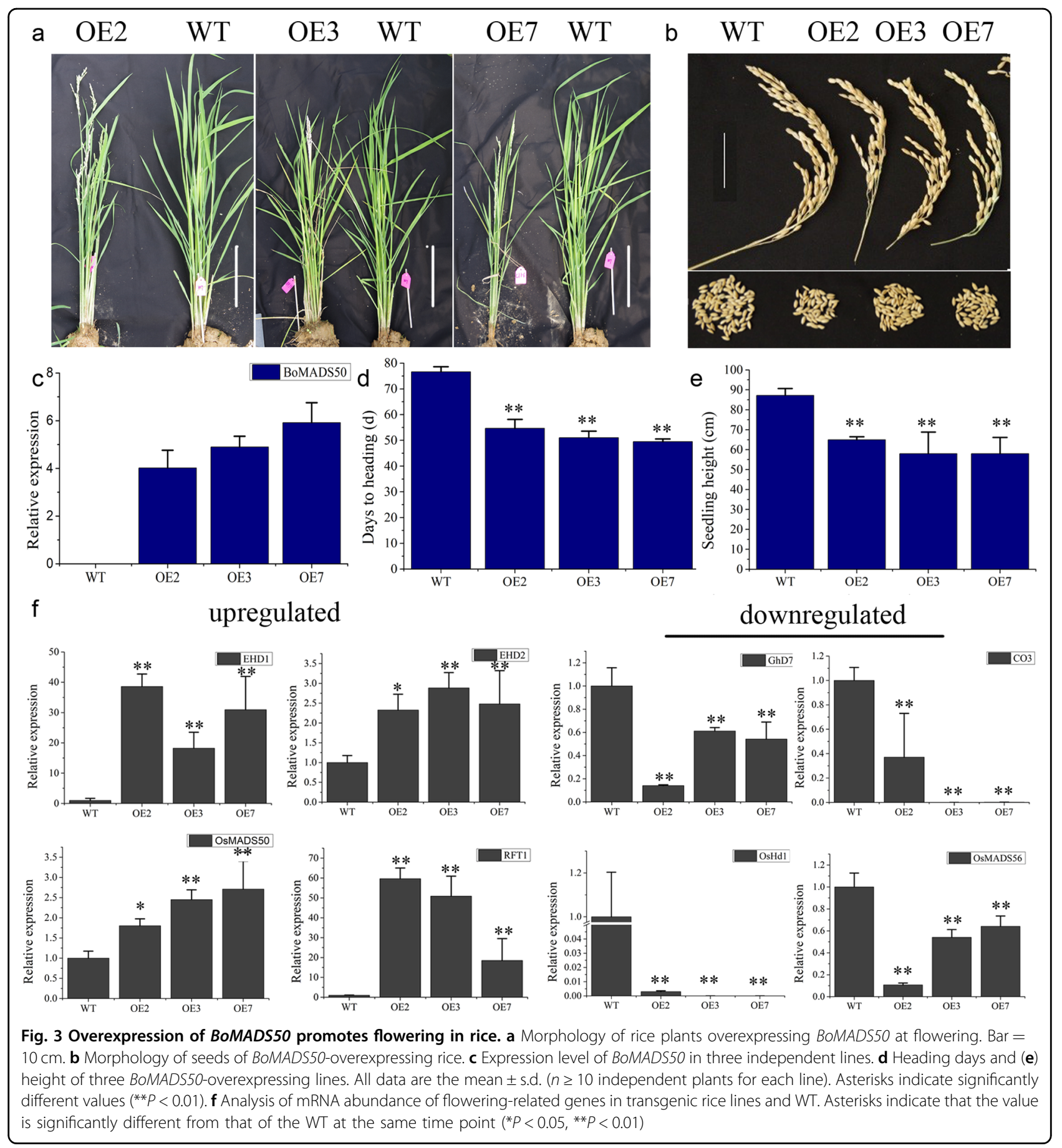

Therefore, ectopic expression of BoMADS50 can promote the flowering of transgenic rice. Further, for confirmation of its role in flowering, BoMADS50 was ectopically overexpressed in the soc1 mutant and WT A. thaliana. As shown in Fig. S2, overexpression of BoMADS50 rescued the late-flowering phenotype of soc1 mutants (Fig. S2a-c) and accelerated flowering in transgenic $A$. thaliana by regulating downstream flowering genes (Fig. S2d-h). Thus, the above results indicate a positive role of BoMADS50 in promoting flowering.

BoMADS50 interacts with AP1/FUL-like proteins in $B$. oldhamii

The APETALA1/FRUITFULL (AP1/FUL)-like genes determine flower meristem characteristics and are key 


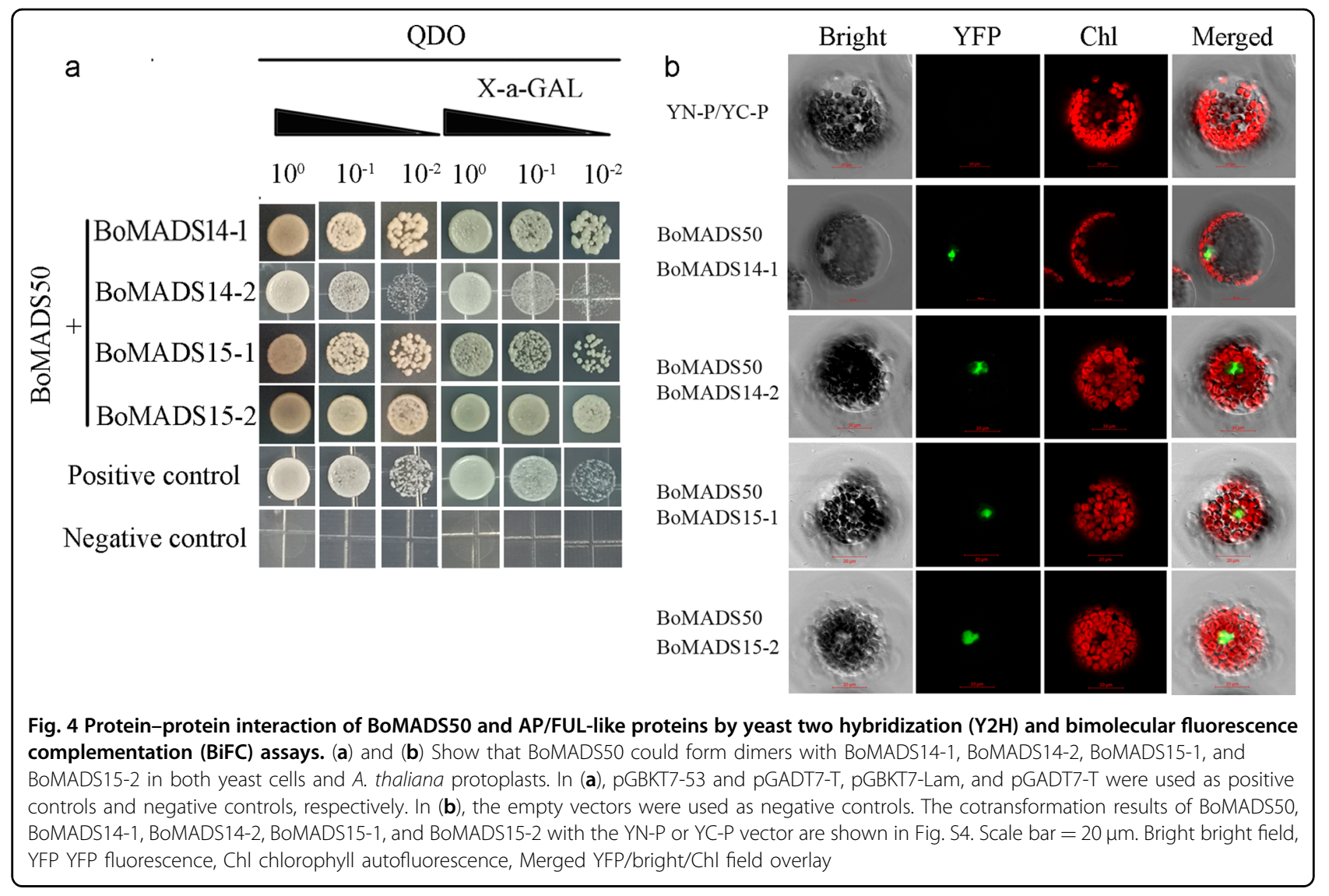

genes for flowering induction and morphology ${ }^{18,19}$. Here, four homologs of OsMADS14 and OsMADS15 were identified and obtained from B. oldhamii (BoMADS14-1, BoMADS14-2, and BoMADS15-1, BoMADS15-2) (Fig. S3a). These genes were highly expressed in the floral II or floral III stage, except for BoMADS14-1, which showed an expression peak in floral I, similar to BoMADS50 (Fig. S3b). This finding suggests that AP1/FUL-like genes may participate in both floret primordium specification and the flower organ development process in B. oldhamii. To test whether BoMADS50 and AP1/FUL proteins interact, we performed yeast two-hybrid $(\mathrm{Y} 2 \mathrm{H})$ experiments. The yeast cells cotransformed with BoMADS50 and BoMADS14-1/2, BoMADS15-1/2, and the positive control grew normally and developed a blue color on -QDO/ $\mathrm{X}$ medium, whereas those with the negative control did not (Fig. 4a). These results suggest that BoMADS50 can form complexes with the four AP1/FUL-like proteins in yeast cells. Bimolecular fluorescence complementation (BiFC) assay was performed in A. thaliana mesophyll protoplast transient expression systems to verify the $\mathrm{Y} 2 \mathrm{H}$ results (Fig. 4b). Except for the negative controls (Figs. 4b and S4), green fluorescence was observed in A. thaliana protoplasts cotransformed with vectors containing BoMADS50 and BoMADS14-1/2 and BoMADS50 and
BoMADS15-1/2. Therefore, both the $\mathrm{Y} 2 \mathrm{H}$ and BiFC assays confirmed the ability of BoMADS50 to heterodimerize with the BoMADS14-1, BoMADS14-2, BoMADS15-1, and BoMADS15-2 proteins in vivo.

\section{BoMADS14-1 can bind to the BoMADS50 promoter and activate its transcription}

Given the similar expression patterns of BoMADS50 and BoMADS14-1 and the putative role of AP1 in activating flowering, we speculated that BoMADS14-1 might be involved in BoMADS50 expression regulation. The promoter of BoMADS50 was cloned and analyzed using the PlantPAN 3.0 database, identifying four CArG motifs located in different clusters (Fig. 5a). The binding affinity of BoMADS14-1 for the BoMADS50 promoter was tested using a yeast one-hybrid $(\mathrm{Y} 1 \mathrm{H})$ assay with a $489 \mathrm{bp}$ fragment, and BoMADS14-1 was shown to bind to the fragment containing a putative CArG motif and activate HIS2 nutritional reporter gene expression (Fig. 5b). Furthermore, we carried out a transient assay to analyze the activating or repressive effect of BoMADS14-1 on the expression of BoMADS50 pro:LUC containing a complete promoter (Fig. 5c). Compared with the coexpression of empty vector as the negative control, that of the fusion protein of BoMADS14-1 triggered and significantly 


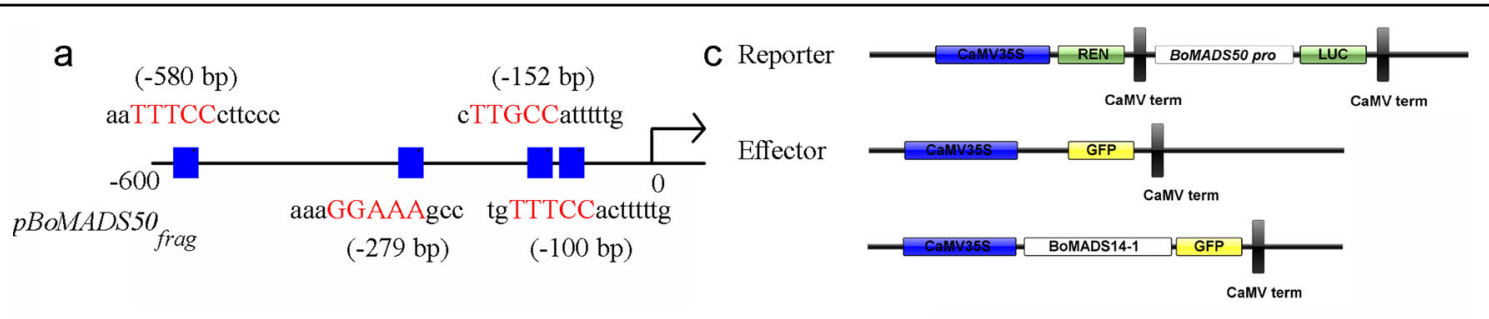

b

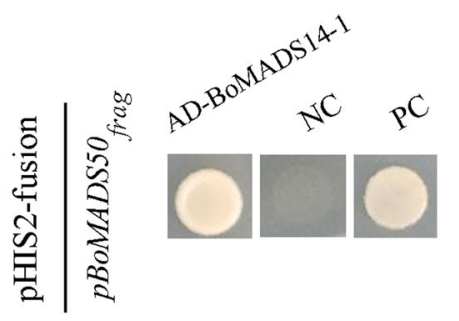

d

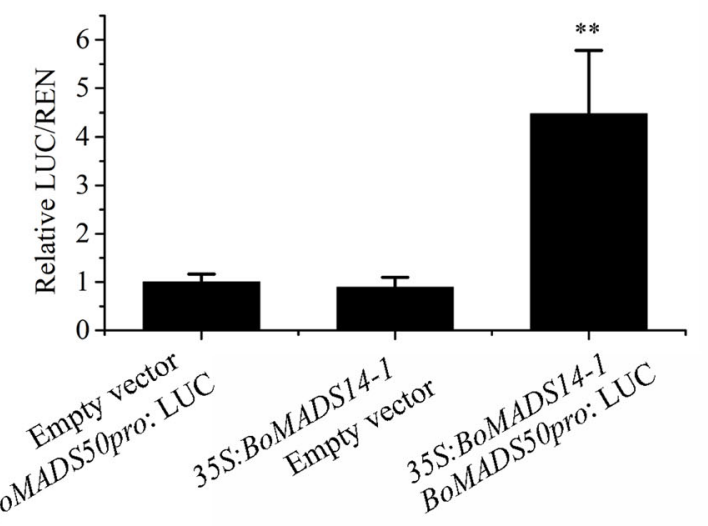

Fig. 5 BoMADS14-1 promotes BoMADS50 expression. a Diagram of the BOMADS50 promoter region containing the putative CArG motif. b BoMADS14-1 bound to the BOMADS50 promoter regions with the putative CArG motif and activated the expression of the nutritional reporter gene HIS2 by yeast one-hybrid assays. c Effector and reporter constructs used in transient dual-luciferase assays. d BoMADS14-1 triggered the expression of BoMAD50pro:LUC with the integration of the 2000-bp BoMADS50 genomic sequence upstream of the ATG before LUC. All data are the mean \pm s.d. $(n \geq 3)$. Asterisks indicate significantly different values $\left({ }^{* *} P<0.01\right)$

upregulated the expression of BoMADS50 pro:LUC (Fig. 5d). The above results suggest that BoMADS14-1 can positively regulate $B O M A D S 50$ expression in vivo.

\section{BoMADS50 and BoMADS50-1 have different regulatory activities}

Our study identified four additional transcripts of BoMADS50 (BoMADS50-1/2/3/4), showing different nucleotide variations. Compared with BoMADS50, BoMADS50-1/2/3/4 had shorter C-terminal regions and various amino acid mutations (Fig. 1a). These sequences belong to an OsMADS50 subgroup and showed high similarities with other OsMADS50-like genes in different bamboo species (Fig. 1b). Ectopic transformation analysis showed that overexpression of BoMADS50-1/2/3/4 could restore the late-flowering phenotypes of $\operatorname{soc} 1 \mathrm{~A}$. thaliana mutants (Fig. S2). Moreover, overexpression of BoMADS50-1/2/3/4 accelerated the flowering of transgenic rice (grown in a greenhouse, Fig. S5), leading to an $\sim 15$-day reduction in flowering time compared with that of the WT (Table S2). Thus, BoMADS50-1/2/3/4 acts as flowering activators.

To determine whether there is functional diversification among different transcripts, we selected BoMADS50-1 for further investigation. BoMADS50-1 was also localized in the nucleus (Fig. 6a). Both $\mathrm{Y} 2 \mathrm{H}$ and BiFC assays showed that BoMADS50-1 could interact with BoMADS50 and form a heterodimer in vivo (Fig. $6 \mathrm{~b}, \mathrm{c}$ ). In bamboo, the $S V P$-like gene can promote flowering in transgenic rice ${ }^{35}$, and in this study, we found that BoSVP has an expression pattern similar to that of BoMADS50 (Fig. S6). This finding indicates that BoSVP functions upstream or downstream of BoMADS50. $\mathrm{Y} 1 \mathrm{H}$ results showed that both BoMADS50 and BoMADS50-1 could bind promoter fragments of itself and BoSVP (Fig. 6d). However, in the dual-luciferase reporter assays, only BoMADS50-1 significantly promoted BoMADS50 and BoSVP expression (Fig. 6e). Moreover, the LUC/REN activity was decreased when BoMADS50-1 and BoMADS50 were cotransformed with target sequences. This result suggests that $B O M A D S 50$ could reduce the binding ability of BoMADS50-1 to itself and downstream targets. Thus, we propose that BoMADS50 and BoMADS50-1 have different regulatory activities.

\section{Discussion}

BoMADS50 acts as a flowering promoter in transgenic $A$. thaliana or rice

Woody bamboos typically exhibit gregarious flowering cycles up to 120 years followed by the death of the parent plants, unique among flowering plants. Although many hypotheses about the bamboo flowering cycle have 


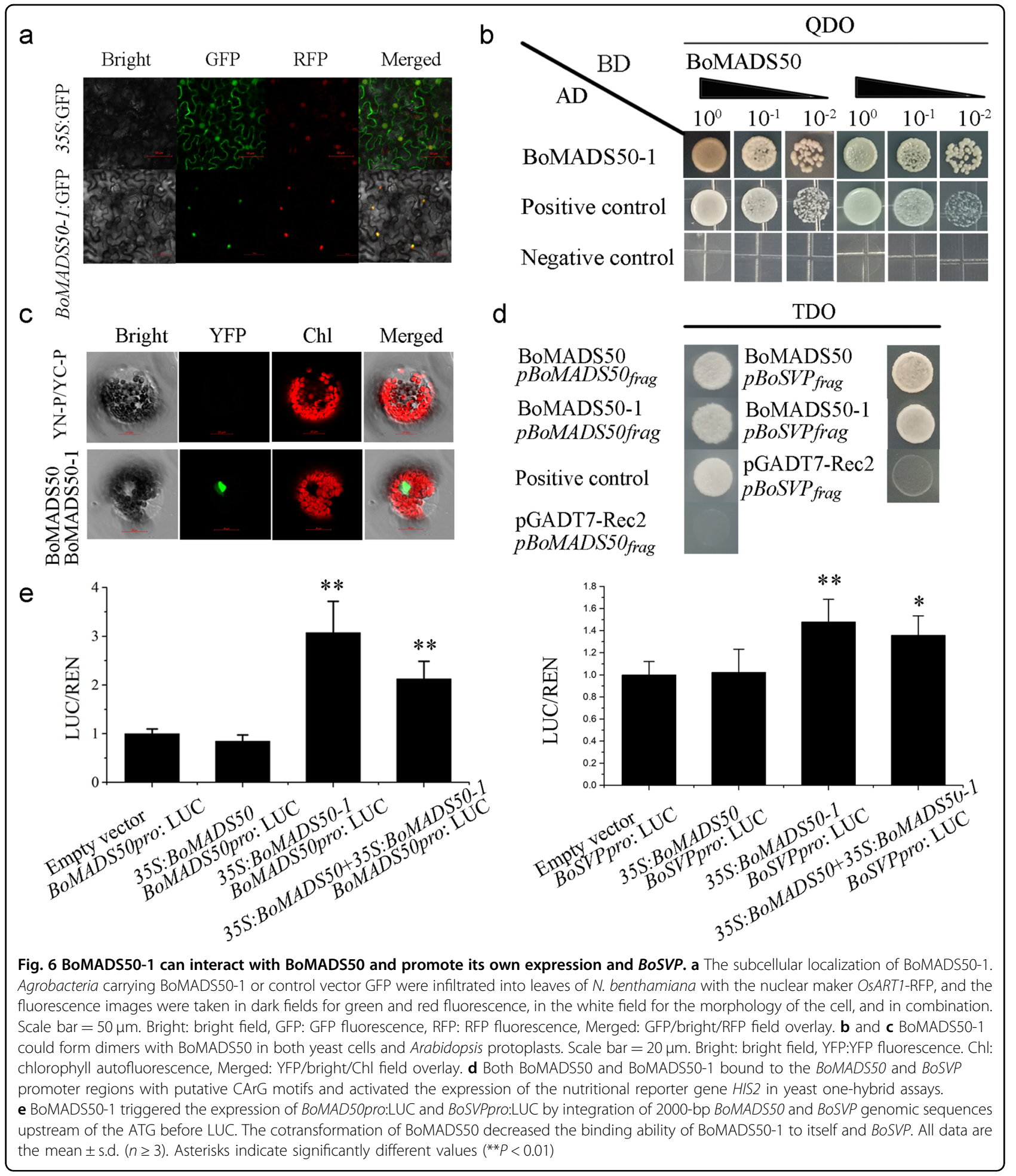

emerged $^{3,4,9}$ and the functions of some flowering genes have been preliminarily analyzed ${ }^{36-38}$, "the molecular aspects of bamboo flowering still remain at a nascent stage $^{\prime 14}$. The SOC1 protein is an essential integrator of multiple flowering signals, regulating the flowering time, floral patterns, and even floral meristem determinacy ${ }^{24,39,40}$. As described by Guo et al. ${ }^{2}$, SOC1/ OsMADS50 orthologs in woody bamboos may be malfunctioning because of protein structure changes or insertions of transposable elements. These malfunctions 
are the potential cause of the extremely long vegetative growth phase in woody bamboos. In this study, a SOC1/ OsMADS50-like gene, BoMADS50, was identified from $B$. oldhamii. However, the MADS-box domain of BoMADS50 was shown to be complete, although a $179 \mathrm{bp}$ interspersed repeat element was inserted in the ORF region of BoMADS50 (Table S3). We further verified the functional role of BoMADS50 by ectopic expression in rice and $A$. thaliana. Similar to $P \nu S O C 1$ from $P$. violascens $^{32}$ and other SOC1-like genes ${ }^{25,41,42}$, BoMADS50 overexpression accelerates flowering in transgenic rice and $A$. thaliana and can rescue the late-flowering phenotype of soc1 mutants (Figs. 3 and S2). In rice, OsMADS5O is an upstream regulator of Ehd1 and RFT1 that regulates flowering under LD conditions ${ }^{28,29}$ but functions either parallel to or downstream of $H d 1$, Ghd7, and Ehd $2^{28,43,44}$. Here, transcript levels of Ehd1 and RFT1 were both upregulated significantly in the BoMADS50overexpressing rice (Fig. 3), indicating that BoMADS50 is involved in an Ehd1-RFT1 pathway to regulate flowering, which is similar to OsMADS50. Interestingly, BoMADS50 overexpression also significantly induced the expression of Ehd2 but repressed that of $H d 1$ or Ghd7 (Fig. 3). This finding suggests that BoMADS50 may be involved in multiple pathways (OsHd1-OsHd3a/RFT1, Ghd7-Ehd1, and Edh2-Ehd1) to affect flowering time in transgenic rice $^{29}$. Thus, in contrast to the previously suggested idea of Guo et al. ${ }^{2}$, BoMADS50 acts as a flowering promoter, and there is no obvious loss or malfunction of BoMADS50 in flowering regulation, at least as shown in transgenic rice or the A. thaliana system.

\section{BoMADS50 may be involved in the flowering regulation of B. oldhamii}

In rice and most species, SOC1-like genes are highly expressed in mature leaves, suggesting their role in the transition from the vegetative to the reproductive phase $^{32,41,43,45,46}$. Moreover, in E. japonica, the expression level of EjSOC1 in early flower buds was significantly higher than that in blooming flowers ${ }^{27}$. Here, expression analysis results showed that BoMADS50 was highly expressed in the floral I stage and mature leaves of $B$. oldhamii (Fig. 2), indicating a potential role of BoMADS50 in flower primordium formation and flowering initiation. However, highly expressed BoMADS5O in the B. oldhamii mature leaves does not induce flowering in this species. This finding may be because the expression of BoMADS50 has not reached the threshold level that can lead to floral initiation. In addition, plant flowering is regulated by a complex gene network ${ }^{29,39,47}$. High expression of BoMADS50 alone may not be sufficient to induce B. oldhamii flowering.

SOC1-like protein is known as an active player by interacting with many other MADS proteins ${ }^{23,24,48}$. For example, SOC1 and AP1/FUL-like proteins can form a network during flowering regulation, and AP1/FUL proteins act downstream or as repressors of SOC1-like genes in model plants ${ }^{24,39,43,47}$. OsMADS14 and OsMADS15 are key AP1/FUL factors involved in flower development and floral initiation regulation in rice ${ }^{18,19,49}$. In this study, the OsMADS14 homologs BoMADS14-1 and BoMADS14-2 and the OsMADS15 homologs BoMADS15-1 and BoMAD15-2 were highly expressed in different flower development stages (Fig. S3), indicating their potential regulatory roles in floral induction and floral meristem determination of $B$. oldhamii. We also found that BoMADS50 can interact with BoMADS14-1/-2 and BoMADS15-1/-2 in both yeast and plant cells, but BoMADS14-1 functioned upstream of BoMADS50 by significantly activating its expression (Figs. 4 and 5 ). Thus, BoMADS50 probably participates in regulating flowering induction by acts synergistically with BoAP1/FUL proteins, and a diversified regulatory relationship between BoMADS50 and BoAP1/FUL might exist in B. oldhamii. The results of heterologous transformation, expression profile, and protein network analysis all imply that BoMADS50 maybe a flowering regulator in B. oldhamii; however, there are still some considerations. The most important reason is that gene functions in a heterologous system may differ from those in the original species. For example, Voogd et al. ${ }^{42}$ showed that overexpression of kiwifruit SOC1-like genes did not cause early flowering in kiwifruit, although their overexpression did in A. thaliana. Therefore, future studies should focus on establishing the exact role of BoMADS50 in transgenic bamboo.

The functional conservatism and specificity of BoMADS50 and BoMADS50-1 might be related to the synchronized and sporadic flowering characteristics of $B$. oldhamii

In this study, four additional transcripts (BoMADS50-1/ 2/3/4) of BoMADS50 were identified. They were clustered with BoMADS50 together in our phylogenetic tree, indicating close evolutionary relationships among them. As shown in a previous study, B. oldhamii belongs to the paleotropical (PTB) woody bamboos ${ }^{50}$, suggesting that multiple copies of the OsMADS50 ortholog might exist in B. oldhamii ${ }^{2}$. However, the ploidy level of $B$. oldhamii has not been determined. Therefore, whether BoMADS50 and BoMADS50-1/2/3/4 are alleles or homeologs has not been confirmed. BoMADS50-1/2/3/4 showed polymorphisms in protein-CDS compared to BoMADS50, but ectopic expression results suggest their conservative roles in promoting flowering (Fig. S5). Interestingly, we found that only BoMADS50-1 could induce the expression of itself and BoSVP (Fig. 6), indicating a different regulatory ability of BoMADS50 and BoMADS50-1. Protein structure analysis showed that BoMADS50-1 had a coding frameshift at the C-terminus due to base variations (Fig. 1). For the full 
function of MADS proteins, the C-terminal motifs are essential and may be responsible for the functional diversification of the major MADS gene subfamilies ${ }^{22}$. Therefore, we speculate that nucleotide variations might endow BoMADS50-1 with a stronger regulatory ability than BoMADS50 by changing the $\mathrm{C}$-terminal structure.

There are many theories about flowering regulation in bamboo $^{3,4,9}$, but many questions remain unanswered. For example, the average flowering time is $\sim 30-60$ years for $B$. oldhamii, but in the early periods of bamboo life, some clumps or small areas of scattered bamboo will blossom sporadically within a B. oldhamii population ${ }^{4,8}$. Franklin ${ }^{3}$ suggested that the term "sporadic flowering" may imply random or other nongregarious patterns of flowering, but there is no convincing evidence that any semelparous bamboo has a reproductive strategy that may be regarded as "not gregarious". Zheng et al. ${ }^{4}$ noted that sporadic flowering or partial flowering may be due to the bamboo forest being composed of different clones, thus forming a flowering wave. However, in Sasa cernua and P. violascens, flowering clumps and nonflowering vegetative clumps were discovered to be of the same clone ${ }^{51,52}$. Therefore, some mechanistic malfunctions or physiological connections probably influence the sporadic flowering of bamboo 9 .

In many species, single-nucleotide polymorphisms (SNPs) are known to be associated with different flowering time genotypes ${ }^{53,54}$. These identifications led us to speculate that the variation in regulatory ability between BoMADS50 and BoMADS50-1 caused by nucleotide variations may be related to the synchronized and sporadic flowering characteristics of $B$. oldhamii. For example, in some flowering clumps, BoMADS50-1 with more robust activity might be selected as a major gene form, leading to different expression levels of downstream flowering genes compared to those of BoMADS50 and resulting in different flowering times. For other vital flowering genes, there may also be different transcripts. The functional conservation of these transcripts could ensure synchronized flowering within a bamboo population, while their potentially different regulatory abilities may cause the sporadic flowering phenotype. It is still challenging to obtain the quantitative loci associated with flowering time due to the unique flowering characteristics of bamboo. To some extent, our results provide a possible hypothesis for the combined extensive synchronized and sporadic flowering phenomenon of woody bamboos.

In summary, based on our results and previous studies in model plants, we propose a hypothetical working model of BoMADS50 in regulating flowering. In addition, BoMADS50-1 was revealed to have a stronger activation ability than BoMADS50, indicating that nucleotide variations may lead to changes in regulatory activity. Above all, $B o M A D S 50$ is probably an important flowering regulator in bamboo. Further, the functional conservatism and specificity of BoMADS50 and BoMADS50-1 may be related to the synchronized and sporadic flowering characteristics in B. oldhamii.

\section{Materials and methods \\ Plant materials and growth conditions}

The flowering tissue culture system is important for woody bamboo flowering mechanistic studies ${ }^{55}$. For $B$. oldhamii, the flowering buds and vegetative shoots originated from the same tissue culture strains ${ }^{56}$. In this study, inflorescences of different developmental periods and different reproductive organs (including pistils, stamens, lemmas, paleas, and lodicules) were derived from these flowered tissue culture plants and have continued flowering since 2001. Moreover, vegetative organs (including roots, stems, immature leaves, and mature leaves) were derived from nonflowering tissue culture plants that had never flowered since their initial culture in $1991^{8}$. All plants were grown under a 16 -h light/8-h dark photoperiod indoors at a temperature of $25-27^{\circ} \mathrm{C}$.

\section{Gene identification and cloning}

cDNA from flower buds in different culture tissue lines in $B$. oldhamii was used as a templet for gene cloning. To isolate a SOC1-like gene from B. oldhamii, we compared the amino acid sequences of $S O C 1$ homologs from grass family plants, including rice, Triticum aestivum, and $Z$. mays, and designed primers (S-F/R) for conserved domain regions. A specific SOC1like cDNA fragment ( $250 \mathrm{bp})$ was obtained, ligated into the pMD18-T vector and confirmed as a partial sequence of a SOC1-like gene of B. oldhamii. The $5^{\prime}$ and $3^{\prime}$ RACE systems were used to isolate the $5^{\prime}$ and $3^{\prime}$ end cDNA with the Rapid Amplification of cDNA Ends kit (Invitrogen, USA). A $330 \mathrm{bp} \mathrm{5'-RACE} \mathrm{cDNA} \mathrm{and} 740 \mathrm{bp}$ 3'-RACE cDNA fragment were spliced to obtain $1049 \mathrm{bp}$ full-length cDNA. With this sequence as the template, the ORF of BoMADS50 was amplified with a high-fidelity enzyme (TaKaRa, Japan). Moreover, four transcripts of BoMADS50 were obtained from B. oldhamii, named BoMADS50-1, BoMADS50-2, BoMADS50-3, and BoMADS50-4. Finally, the nucleotide variations were further confirmed by PCR using DNA templates of different lines. The primers used for BoMADS50 cloning are shown in Table S1.

\section{Sequence alignment and phylogenetic analysis}

For phylogenetic analysis, the SOC1- or AP1-like proteins in rice or A. thaliana were downloaded from the Rice Genome Annotation Project database (http://rice. plantbiology.msu.edu/) or The Arabidopsis Information Resource database (https://www.arabidopsis.org/), 
respectively. For identification of SOC1- or AP1-like proteins in different bamboo species, the amino acid sequences of OsMADS50, OsMADS56, and OsMADS60 (SOC1-like), OsMADS14, OsMADS15, OsMADS18, and OsMADS20 (AP1-like) from rice were used as query sequences to blast against the moso bamboo protein V2 database $^{11}$, Bonia amplexicaulis, Olyra latifolia, Guadua angustifolia, Raddia guianensi protein databases ${ }^{2}$ and $B$. edulis database ${ }^{57}$ with an $e$-value cutoff of $10^{-50}$. Additionally, the amino acid sequence of PvSOC1 in P. violascens was obtained from $\mathrm{Ma}^{58}$, and the GenBank accession number of PvMADS56 is AQS27937.1 ${ }^{31}$. Multiple sequence alignment was performed using ClustalX 2.0 , and the phylogenetic tree was constructed with MEGA 7.0 using the maximum-likelihood (ML) method with 1000 bootstrap replicates.

\section{Expression profile revealed by qRT-PCR}

Total RNA was extracted from flowers in bamboo, leaves in transgenic $A$. thaliana, and rice using TRIzol reagent. Single-stranded DNA was synthesized using the PrimeScript $^{\mathrm{TM}} \mathrm{RT}$ reagent Kit with gDNA Eraser (TaKaRa) according to the instructions. The transcripts of BoMADS50, BoMADS14-1, BoMADS14-2, BoMADS15-1, and BoMADS15-2 in bamboo and the key flowering genes in transgenic plants were assessed using qRT-PCR with specific primers. In $B$. oldhamii, NTB was used as a reference gene ${ }^{59}$, while in $A$. thaliana and rice, Atactin or $O s U b q$ was used as a reference gene. The primers used for gene expression analysis are listed in Table S1. qPCR was performed using SYBR Green Master Mix (Bio-Rad) on a real-time PCR instrument (Bio-Rad). All qPCR assays were performed with three biological and four technical replicates, and the $2^{-\triangle \Delta C T}$ method was used for quantitative analysis. For BoMADS50 in transgenic rice or $A$. thaliana lines, the expression of the endogenous homologous genes OsMADS50 or SOC1 was normalized to 1 . For other flowering genes in rice and A. thaliana, their relative expression in the WT was normalized to 1 .

\section{Subcellular localization}

For the subcellular localization assay, the CDSs of BoMADS50 and BoMADS50-1 were cloned into the pCAMBIA-1300-GFP vector to generate 35S: BoMADS50:GFP and 35S:BoMADS50-1:GFP. The empty vector was transformed as a negative control, and nuclear-localized OsART1 served as a nuclear localization marker by inducing each transfection ${ }^{60}$. Transient transformation of tobacco leaves was performed as described by Yang et al. ${ }^{61}$. The transformed plants were then cultured in the dark for more than $48 \mathrm{~h}$, followed by GFP fluorescence detection under a confocal microscope (Zeiss, LSM 880) with both 488 and $594 \mathrm{~nm}$ argon lasers.

\section{Yeast two-hybrid (Y2H) assay}

The CDS of BoMADS50 was cloned into the pGBKT-7 vector (Clontech, Beijing, China), and the CDSs of putative interacting genes (BoMADS50-1, BoMADS14-1, BoMADS14-2, BoMADS15-1 and BoMADS15-2) were cloned into pGADT7 vectors. pGBKT7-BoMADS50 was cotransformed with the corresponding pGADT7 recombinant plasmid into the yeast strain AH109 following the manufacturer's instructions for the Matchmaker GAL4 two-hybrid system (Clontech). pGBKT-53+pGADT7-T and pGBKT53+pGADT-Lam were used as positive and negative controls, respectively. The transformed yeast was cultured in $\mathrm{SD} /$-Trp/-Leu (-DDO) media at $30^{\circ} \mathrm{C}$ for 3 days, and a serial decimal dilution was used for the spot assay on dropout media SD/-Trp/-Leu/-His/-Ade (-QDO) and $-\mathrm{QDO} / \mathrm{X}-\mathrm{a}-\mathrm{Gal}(-\mathrm{QDO} / \mathrm{X})$. The protein interactions were confirmed for $\sim 3-5$ days at $30^{\circ} \mathrm{C}$.

\section{BiFC assay}

The CDS of BoMADS50 was cloned into the pSAT1nEYFP-C1 (YN-P) vector. The other genes of interest were cloned into the pSAT4-cEYFP-C1-B (YC-P) vector. All empty vectors were used as a negative control, while the recombinant plasmids (YN-BoMADS50, BoMADS501-YC, BoMADS14-1-YC, BoMADS14-2-YC, BoMADS151-YC, BoMADS15-2-YC) were also cotransformed with the YN-P or YC-P vector to exclude false positives. These constructs were transiently expressed in $A$. thaliana mesophyll protoplasts, according to a previous report ${ }^{62}$. The transfected cells were imaged using a confocal microscope at wavelengths of $488 \mathrm{~nm}$ and a $594 \mathrm{~nm}$ argon laser. The combinations of BiFC were performed in at least three biological replicates.

\section{$\mathrm{Y} 1 \mathrm{H}$ assay}

We cloned the CDSs of BoMADS50, BoMADS50-1, and BoMADS14-1 into the vector pGADT7-Rec and the 489bp BoMADS50 promoter fragment and 416-bp BoSVP promoter fragment containing the putative CArG motif into the vector pHIS2. The yeast strain Y187 was cotransformed with the corresponding pGADT7 and pHIS2 constructs following the manufacturer's instructions for the yeast one-hybrid library screening system (Clontech). pHIS53 + pGADT753 was used as a positive control, and pHIS-proBoMADS50 $0_{\mathrm{frag}}+$ pGADT7-Rec and pHIS-proBoSVP $P_{\text {frag }}+$ pGADT7-Rec were used as negative controls. The transformed strain was cultured at $30^{\circ} \mathrm{C}$ for 2-3 days on SD/-Trp/-Leu media, and serial decimal dilution was used for spot assays on $\mathrm{SD} /-\mathrm{Trp} /-\mathrm{Leu} /$-His media with $50 \mathrm{mM} 3$-AT.

\section{Dual-luciferase reporter assay}

The BoMADS50 and BoSVP promoters (2000 bp genomic sequence before ATG) were amplified and cloned 
into the pGreenII 0800-LUC vector, which generated the reporter construct BoMADS50pro:LUC or BoSVPpro: LUC. The effectors were constructed by cloning the openreading frames into the pCAMBIA1300 vector with Flag driven by the CaMV $35 \mathrm{~S}$ promoter (35S:BoMADS50, 35S: BoMADS50-1, 35S:BoMADS14-1). The empty vectors were coexpressed with reporter or effector as a negative control. The transformation of all the effectors and reporters followed the protocol of ${ }^{16}$. Agrobacterium tumefaciens was cultured overnight and resuspended to $\mathrm{OD}_{600}$ values of $0.6-0.8$. The mixture with the effector and reporter strain at a ratio of 9:1 was resuspended in infiltration media (10 mM MES, $1 \mathrm{M} \mathrm{MgCl}_{2}, 200 \mu \mathrm{M}$ acetosyringone) and incubated for $3-4 \mathrm{~h}$ at room temperature without shaking. The infiltrated young Nicotiana benthamiana plants were incubated for more than $48 \mathrm{~h}$ under weak light conditions. At least four biological replicates were performed for each sample. The LUC/ REN signals were calculated using dual-LUC assay solutions (Promega) on a luminometer (Promega). Finally, the LUC/REN value of the control group (coexpressed with empty vector and BoMADS50pro:LUC; empty vector and BoSVPpro:LUC) was normalized to 1.

\section{Vector construction and generation of BoMADS50- overexpressing $A$. thaliana and rice}

The recombinant plasmids $p$ CAMBIA1300-BoMADS50 and $p C A M B I A 1300-B o M A D S 50-1 / 2 / 3 / 4$ were introduced into A. tumefaciens GV3101 and EHA105, respectively. The floral dip method was used for A. thaliana transformation, and an Agrobacterium-mediated rice transformation method was described by Nishimura et al. ${ }^{63}$. Regenerated seedlings were selected on MS medium with $50 \mathrm{mg} \mathrm{L}^{-1}$ hygromycin. The expression levels of BoMADS50 in transgenic WT A. thaliana (Columbia), soc1 mutant (SALK 138131C, Columbia) and rice (O. sativa L. subsp. japonica cv. zhonghua 11) plants were analyzed by RT-PCR and confirmed using qRT-PCR. Homozygous transgenic lines of the T3 generation were used for further analyses. For phenotypic observation, transgenic $A$. thaliana seedlings were grown in a greenhouse with 16 -h light $/ 8$-h dark at $21-23^{\circ} \mathrm{C}$, and transgenic rice seedlings were planted in a growth room owith14-h light $/ 10$-h dark at $25-30^{\circ} \mathrm{C}$. All the samples used for gene expression detection were collected in the morning ( $~ 5-6 \mathrm{~h}$ after the light was turned on) based on a previous study by Lee et $\mathrm{al}^{43}$.

\section{Acknowledgements}

We thank Dr. Jin Zhang and Dr. Naresh Vasupalli for polishing the language of this study. This work was supported by grants from the Natural Science Foundation of Zhejiang Province (LZ20C160002), the National Natural Science Foundation of China (31971735), and the State Key Laboratory of Subtropical Silviculture (ZY20180203).

\section{Author details}

${ }^{1}$ State Key Laboratory of Subtropical Silviculture, Zhejiang A \& F University, Lin'an, 311300 Hangzhou, China. 'State Key Laboratory for Conservation and Utilization of Subtropical Agro-bioresources, South China Agricultural University, 510642 Guangzhou, China. ${ }^{3}$ Guangdong Key Laboratory for Innovative Development and Utilization of Forest Plant Germplasm, College of Forestry and Landscape Architecture, South China Agricultural University, 510642 Guangzhou, China

\section{Author contributions}

X.C.L. and A.M.W. designed the research. D.H. and L.L. mainly performed the research. T.F.M., J.L.P., and Z.Y.Z. finished some parts of the experiments. D.H. wrote the manuscript. M.Z.L., X.C.L., and A.M.W. revised and approved the manuscript.

\section{Conflict of interest}

The authors declare no competing interests.

Supplementary information The online version contains supplementary material available at https://doi.org/10.1038/s41438-021-00557-4.

Received: 15 August 2020 Revised: 16 March 2021 Accepted: 26 March 2021

Published online: 01 June 2021

\section{References}

1. Fang, W. et al. Chinese Economic Bamboo (Science Press, 2015).

2. Guo, Z. H. et al. Genome sequences provide insights into the reticulate origin and unique traits of woody bamboos. Mol. Plant 12, 1353-1365 (2019).

3. Franklin, D. C. Synchrony and asynchrony: observations and hypotheses for the flowering wave in a long-lived semelparous bamboo. J. Biogeogr. 31, 773-786 (2010).

4. Zheng, X., Lin, S. Y., Fu, H. J., Wan, Y. W. \& Ding, Y. L. The bamboo flowering cycle sheds light on flowering diversity. Front. Plant Sci. 11, 381 (2020).

5. Guerreiro, C. Flowering cycles of woody bamboos native to southern South America. J. Plant Res. 127, 307-313 (2014).

6. Lin, S. Y. \& Mao, G. X. The habit and regeneration of bamboo flowering. For. Sci. Technol. 32, 23-25 (2007).

7. Zeng, H. Y., Lu, Y. T., Yang, X. M., Xu, Y. H. \& Lin, X. C. Ectopic expression of the BoTFL1-like gene of Bambusa oldhamii delays blossoming in Arabidopsis thaliana and rescues the tfl1 mutant phenotype. Genet. Mol. Res. 14, 9306-9317 (2015).

8. Lin, X. C. et al. Understanding bamboo flowering based on large-scale analysis of expressed sequence tags. Genet. Mol. Res. 9, 1085-1093 (2010).

9. Suyama, Y., Suzuki, J. I. \& Makita, A. For the comprehension of gregarious flowering in bamboos. Jpn. J. Ecol. 60, 97-106 (2010).

10. Peng, Z. H. et al. The draft genome of the fast-growing non-timber forest species moso bamboo (Phyllostachys heterocycla). Nat. Genet. 45, 456-461 (2013).

11. Zhao, H. S. et al. Chromosome-level reference genome and alternative splicing atlas of moso bamboo (Phyllostachys edulis). Gigascience 7, 1-12 (2018).

12. Gao, J., Zhang, Y., Zhang, C. L., Qi, F. Y. \& Li, X. P. Characterization of the floral transcriptome of Moso bamboo (Phyllostachys edulis) at different flowering developmental stages by transcriptome sequencing and RNA-seq analysis. PLOS ONE 9, e98910 (2014).

13. Xiao, G. H. et al. Overexpression of PvCO1, a bamboo CONSTANS-LIKE gene delays flowering by reducing expression of the FT gene in transgenic Arabidopsis. BMC Plant Biol. 18, 232 (2018).

14. Biswas, P., Chakraborty, S., Dutta, S., Pal, A. \& Das, M. Bamboo flowering from the perspective of comparative genomics and transcriptomics. Front. Plant Sci. 7, 1900 (2016).

15. Liu, J. et al. MIKC C-type MADS-box genes in Rosa chinensis: the remarkable expansion of $A B C D E$ model genes and their roles in floral organogenesis. Hortic. Res. 5, 25 (2018).

16. Tao, J. H., Liang, W. Q., An, G. H. \& Zhang, D. B. OsMADS6 controls flower development by activating rice FACTOR OF DNA METHYLATION LIKE1. Plant Physiol. 177, 713-727 (2018). 
17. Véronique, $H$. et al. Tetramerization of MADS family transcription factors SEPALLATA3 and AGAMOUS is required for floral meristem determinacy in Arabidopsis. Nucleic Acids Res. 46, 4966-4977 (2018).

18. Wu, F. et al. The ABCs of flower development: mutational analysis of AP1/FULlike genes in rice provides evidence for a homeotic (A)-function in grasses. Plant J. 89, 310-324 (2016)

19. Lu, S. J. et al. Overexpression of a transcription factor OsMADS15 modifies plant architecture and flowering time in rice (Oryza sativa L.). Plant Mol. Biol. Report. 30, 1461-1469 (2012)

20. Mateos, J. L., Tilmes, V., Madrigal, P., Severing, E. \& Coupland, G. Divergence of regulatory networks governed by the orthologous transcription factors FLC and PEP1 in Brassicaceae species. Proc. Natl Acad. Sci. USA 114, E11037-E11046 (2017).

21. Ruokolainen, S. et al. Characterization of SQUAMOSA-like genes in Gerbera hybrida, including one involved in reproductive transition. BMC Plant Biol. 10, 128 (2010).

22. Vandenbussche, M., Theissen, G., Peer, Y. V. \& Geratsa, T. Structural diversification and neo-functionalization during floral MADS-box gene evolution by C-terminal frameshift mutations. Nucleic Acids Res. 31, 4401-4409 (2003).

23. Hyun, Y. et al. Multi-layered regulation of SPL15 and cooperation with SOC1 integrate endogenous flowering pathways at the Arabidopsis shoot meristem. Dev. Cell 37, 254-266 (2016).

24. Immink, R. G. et al. Characterization of SOC1's central role in flowering by the identification of its upstream and downstream regulators. Plant Physiol. 160 433-449 (2012).

25. Zhao, S. Z. et al. ZmSOC1, a MADS-box transcription factor from Zea mays, promotes flowering in Arabidopsis. Int. J. Mol. Sci. 15, 19987-20003 (2014).

26. Fudge, J. B., Lee, R. H., Laurie, R. E., Mysore, K. S. \& Wen, J. Medicago truncatula SOC1 genes are up-regulated by environmental cues that promote flowering. Front. Plant Sci. 9, 496 (2018)

27. Jiang, Y. Y. et al. The role of EjSOC1s in flower initiation in Eriobotrya japonica. Front. Plant Sci. 4, 253 (2019).

28. Komiya, R., Yokoi, S. \& Shimamoto, K. A gene network for long-day flowering activates RFT1 encoding a mobile flowering signal in rice. Development 136, 3443-3450 (2009).

29. Lee, Y. S. \& An, G. Regulation of flowering time in rice. J. Plant Biol. 58, 353-360 (2015).

30. Rao, N. N., Prasad, K., Kumar, P. R. \& Vijayraghavan, U. Distinct regulatory role for RFL, the rice LFY homolog, in determining flowering time and plant architecture. Proc. Natl Acad. Sci. USA 105, 3646-3651 (2008).

31. Liu, S. N. et al. Ectopic expression of a SOC1 homolog from Phyllostachys violascens alters flowering time and identity of floral organs in Arabidopsis thaliana. Trees 30, 2203-2215 (2016a).

32. Liu, S. N., Ma, T. F., Ma, L. \& Lin, X. C. Ectopic expression of PvSOC1, a homolog of SOC1 from Phyllostachys violascens, promotes flowering in Arabidopsis and rice. Acta Physiol. Plant. 38, 166 (2016b).

33. Lin, S. Y. et al. Research on inflorescence establishment and revision of inflorescence type in bamboo plants. J. Nanjing For. Univ. (Nat. Sci. Ed.) 42, 5-10 (2018b)

34. Lin, S. Y. et al. The research on the anther development and floral morphology characteristics of Bambusa oldhami 'Xia Zao' ZSX. J. Nanjing For. Univ. (Nat. Sci. Ed.) 43, 11-17 (2018a)

35. Liu, S. N. Cloning and Functional Analysis of SVP-like and SOC1-like Genes from Phyllostachys violascens[D] (Beijing Forestry University, 2016).

36. Liu, S. N., Li, X. F., Yang, H. \& Lin, X. C. Ectopic expression of BoYAB1, a member of YABBY gene family in Bambusa oldhamii, causes leaf curling and late flowering in Arabidopsis thaliana. J. Hortic. Sci. Biotechnol. 95, 169-174 (2019).

37. Zheng, Z. G. et al. Overexpression of PvPin1, a bamboo homolog of PIN1-type Parvulin 1, delays flowering time in transgenic Arabidopsis and rice. Front. Pant Sci. 8, 1526 (2017).

38. Zhu, L. F. et al. Functional analysis of Pl-like gene in relation to flower development from bamboo (Bambusa oldhamii). J. Genet. 95, 71-78 (2016).

39. Lee, J. \& Lee, I. Regulation and function of SOC1, a flowering pathway integrator. J. Exp. Bot. 61, 2247-2254 (2010).

40. Li, C. et al. Analysis of interactions between AGL18 family members and flowering time integrator factor SOC1 in Brassica juncea. Acta Hortic. Sin. $\mathbf{4 4}$ 463-474 (2017).
41. Shitsukawa, N., Ikari, C., Mitsuya, T., Sakiyama, T. \& Murai, K. Wheat SOC1 functions independently of WAP1NRN1, an integrator of vernalization and photoperiod flowering promotion pathways. Physiol. Plant. 130, 627-636 (2007).

42. Voogd, C., Wang, T. \& Varkonyi-Gasic, E. Functional and expression analyses of kiwifruit SOC1-like genes suggest that they may not have a role in the transition to flowering but may affect the duration of dormancy. J. Exp. Bot. 66, 4699-4710 (2015).

43. Lee, S., Kim, J., Han, J. J., Han, M. J. \& An, G. Functional analyses of the flowering time gene OSMADS50, the putative SUPPRESSOR OF OVEREXPRESSION OF CO 1/AGAMOUS-LIKE 20 (SOC1/AGL20) ortholog in rice. Plant J. 38, 754-764 (2004).

44. Ryu, C. H. et al. OsMADS50 and OsMADS56 function antagonistically in regulating long day (LD)-dependent flowering in rice. Plant Cell Environ. 32, 1412-1427 (2009).

45. Lee, $\mathrm{H}$. et al. The AGAMOUS-LIKE 20 MADS domain protein integrates floral inductive pathways in Arabidopsis. Genes Dev. 14, 2366-2376 (2000).

46. Zhang, Y. X. et al. Isolation and characterization of a SOC1-Like gene from tree peony (Paeonia suffruticosa). Plant Mol. Biol. Report. 33, 5 (2015).

47. Smaczniak, C. et al. Characterization of MADS-domain transcription factor complexes in Arabidopsis flower development. Proc. Natl. Acad. Sci. USA 109 1560-1565 (2012).

48. Chen, J. et al. Promoter isolation of flowering signal integrator SOC1 gene and its interactions with FLC and SVP proteins in Brassica juncea. Acta Hortic. Sin. 42, 1931-1943 (2015).

49. Jeon, J. S. et al. Production of transgenic rice plants showing reduced heading date and plant height by ectopic expression of rice MADS-box genes. Mol. Breed. 6, 581-592 (2000).

50. Wysocki, W. P., Clark, L. G., Attigala, L., Ruiz-Sanchez, E. \& Duvall, M. R. Evolution of the bamboos (Bambusoideae; Poaceae): a full plastome phylogenomic analysis. BMC Evolut. Biol. 15, 50 (2015).

51. Jiao, Y. L. et al. Comparative transcriptomic analysis of the flower induction and development of the Lei bamboo (Phyllostachys violascens). BMC Bioinforma. 20, Suppl 25 (2019).

52. Keiko, K. \& Takayuki, K. Estimation of outcrossing rates at small-scale flowering sites of the dwarf bamboo species, Sasa cernua. J .Plant Res. 124, 683-688 (2011).

53. Rui, M. I., Jia, J. Z. \& Han, D. J. SNP analysis of autonomous pathway related gene TaFLD of flowering time in wheat. J. Triticeae Crops 31, 9-14 (2011).

54. Takeshima, R. et al. A soybean quantitative trait locus that promotes flowering under long days is identified as FT5a, a FLOWERING LOCUS T ortholog. J. Exp. Bot. 67, 5247-5258 (2016).

55. Yuan, J. L., Yue, J. J., Gu, X. P. \& Lin, C. S. Flowering of woody bamboo in tissue culture systems. Front. Plant Sci. 8, 1589 (2017).

56. Huang, L. C., Huang, B. L. \& Chen, W. L. Tissue culture investigations of bamboo-IV. Organogenesis leading to adventitious shoots and plants in excised shoot apices. Environ. Exp. Bot. 29, 307-315 (1989).

57. Shih, M. C. et al. BeMADS1 is a key to delivery MADSs into nucleus in reproductive tissues-De novo characterization of Bambusa edulis transcriptome and study of MADS genes in bamboo floral development. BMC Plant Biol. 14, 179-179 (2014).

58. Ma, T. F. Molecular Cloning and Functional Analysis of BoSOC1 in Bambusa oldhamii and PvSOC1 in Phyllostachys violascens[D] (Zhejiang Agriculture and Forestry University, 2013).

59. Fan, $C$. et al. Selection of reference genes for quantitative real-time PCR in bamboo (Phyllostachys edulis). PLoS ONE 8, e56573 (2013).

60. Yamaji, $N$. et al. A zinc finger transcription factor ART1 regulates multiple genes implicated in aluminum tolerance in rice. Plant Cell 21, 3339-3349 (2009).

61. Yang, Y., Li, R. \& Min, Q. In vivo analysis of plant promoters and transcription factors by agroinfiltration of tobacco leaves. Plant J. 22, 543-551 (2000).

62. Yoo, S. D., Cho, Y. H. \& Sheen, J. Arabidopsis mesophyll protoplasts: a versatile cell system for transient gene expression analysis. Nat. Protoc. 2, 1565-1572 (2007).

63. Nishimura, A., Aichi, I. \& Matsuoka, M. A protocol for Agrobacterium-mediated transformation in rice. Nat. Protoc. 1, 2796-802 (2006). 